\title{
Konjugatimpfstoff in höherem Alter immunogen
}

Fragestellung: Welchen Effekt hat die Pneumokokkenimpfung, wenn es ums Verhindern der Pneumonie bei $\geq 65$-Jährigen geht?

Hintergrund: Die Leistungsfähigkeit des Immunsystems nimmt mit zunehmendem Alter ab, unabhängig von bestehenden Komorbiditäten. Daher ist die Antikörperantwort nach einer Impfung mit dem Polysaccharid-Impfstoff PPSV-23 im Alter verglichen mit einem Kollektiv jüngerer Menschen niedriger. Der Konjugatimpfstoff PCV-13 induziert eine durch T-Zellen vermittelte Immunantwort, was auch bei Älteren effektiver sein könnte.

\section{Originalie}

Van Werkhoven $\mathrm{CH}$, Huijts SM, Bolkenbaas $\mathrm{M}$ et al. The Impact of Age on the Efficacy of 13-valent Pneumococcal Conjugate Vaccine in Elderly. Clin Infect Dis. 2015 Dec 15;61(12):1835-8
Patienten und Methoden: Mit dem Fokus auf Multimorbidität und Immunseneszenz wurde in dieser Posthoc-Analyse die Effektivität der Pneumokokkenimpfung mit dem 13-valenten Pneumokokken-Konjugat-Impfstoff bei über 65-Jährigen untersucht. Basis der Analyse ist die Studie „Polysaccharide conjugate vaccine against pneumococcal pneumonia in adults" $[1,2]$. Eine rezente Studie mit 84.496 Probanden im Alter $\geq 65$ Jahren und einer Beobachtungszeit von 3,97 Jahren. Die Diagnose „Pneumonie“ wurde nach den bekannten Community-acquired-Pneumonia-(CAP-)Kriterien gestellt. Zusätzlich wurde die Diagnose der Pneumonie mit dem Urin-Antigen-Test auf Pneumokokken gesichert. Dieser war bei den Unter-70-jährigen zu 93\% positiv, bei den Über80 -jährigen zu etwa $90 \%$.

Ergebnisse: In der Gruppe der geimpften 65- bzw. 75-jährigen kam es zu einer Reduzierung der CAP und invasiven Pneumokokken-Erkrankung von 65 auf 40\%. Das beste Ansprechen auf die Impfung mit PCV-13 zeigte sich in der Gruppe der $\geq 65$-Jährigen. 2941 Patienten waren in dieser Analyse älter als 85 Jahre.

Schlussfolgerungen: Die Studienergebnisse zeigen, dass der Konjugatimpfstoff eine hohe Immunogenität hat. Die Effektivität nimmt mit zunehmend höherem Lebensalter zwar ab, bietet aber immer noch einen guten serotypspezifischen Schutz vor nicht invasiver Pneumonie.

\section{- Kommentar von Prof. Dr. med. Hans Jürgen Heppner}

\section{Pneumonien sind für Ältere besonders fatal, Impfung ist zu empfehlen}

Die Immunseneszenz führt zu verringertem Ansprechen auf (neue) Antigene, kann zu einer eingeschränkten humoralen Immunantwort führen und zieht eine höhere Mortalität und Morbidität im Alter nach sich [3]. Funktionalität und Multimorbidität spielen eine große Rolle bei der Einschätzung der Immunkompetenz geriatrischer Patienten [4]. Da Pneumokokken die häufigsten Erreger der nicht invasiven Pneumonie bei alten Patienten sind [5], ist die Impfung eine unumgängliche präventive Maßnahme, um die Morbidität und die Letalität in diesem Personenkreis zu senken. Die ambulant erworbene Pneumonie (CAP) muss hier als Maß für die Impfeffektivität angesehen werden.

Eine erfolgreiche Strategie hat die CAPITA-Studie gezeigt [1], in der durch die Impfung mit dem 13-valenten Konjugatimpfstoff die Rate invasiver und nicht invasiver Pneumonien gesenkt wurde. Die höhere Immunogenität des 13-valenten Konjugat-Impfstoffes im Vergleich zum nicht konjugierten Polysaccharid-Impfstoff wurde bereits beschrieben [6]. Es muss berücksichtigt werden, dass geriatrische Patienten nach einer Pneumonie oft deutliche Funktionseinschränkungen und Verluste in ihrer Alltagskompetenz behalten [7]. Daher haben die Deutschen Gesellschaft für Pneumologie und Beatmungsmedizin, die Deutschen Gesellschaft für Infektiologie, die Paul-Ehrlich-Gesellschaft für Chemotherapie, die CAPNETZ-Stiftung sowie die Österreichischen und Schweizerischen Gesellschaften für Pneumologie von 2015 die Impfung mit dem konjugierten Pneumokokken-Impfstoff PCV13 in ihre Empfehlungen aufgenommen. Ebenso tat dies die American
Geriatric Society (AGS) in ihrer Leitlinie [8]. Die Ständige Impfkommission (STIKO) hat den Impfstoff zwar für bestimmte Fälle in die Empfehlungen für Über-65-Jährige aufgenommen, empfiehlt jedoch trotzdem generell den Polysaccharidimpfstoff [9].

\footnotetext{
Literatur:

1. Bonten MJ, et al. N Engl J Med. 2015 Mar 19;372(12):1114-25.

2. van Werkhoven CH, Bonten MJ. Future Microbiol. 2015 Sep;10:1405-13.

3. Pletz MW et al. J Neuroimmunol 2003;137:1-11.

4. Kwetkat A et al. Impfungen im Alter. Pneumologe 2014; 11:478-85.

5. Torres A et al. Eur J Clin Microbiol Infect Dis 2014:33:1065-79.

6. de Roux A, et al. Clin Infect Dis 2008:46(7):1015-23.

7. Davydow DS et al. Am J Med 2013;126,615-24 e615.

8. Pilkinton MA, Talbot KH, J Am Geriatr Soc 2015;63:584-88.

9. RKI, Epidemiologisches Bulletin Nr. 34, August 2015
}

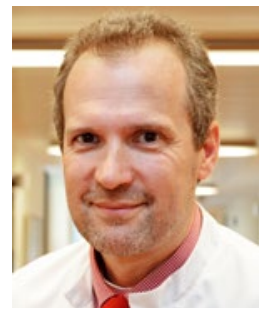

Prof. Dr. med. Hans Jürgen Heppner

Chefarzt Geriatrische Klinik und Tagesklinik Lehrstuhl für Geriatrie

Universität Witten/Herdecke

Dr.-Moeller-Straße 15, 58332 Schwelm hans-juergen.heppner@helios-kliniken.de hans.heppner@uni-wh.de 